\title{
\begin{tabular}{l|l} 
MitTraries & DSpace@MIT
\end{tabular}
}

\author{
MIT Open Access Articles
}

Droplets bouncing on a wet, inclined surface

The MIT Faculty has made this article openly available. Please share how this access benefits you. Your story matters.

Citation: Gilet, Tristan, and John W. M. Bush. "Droplets bouncing on a wet, inclined surface." Physics of Fluids 24, no. 12 (2012): 122103. (C) 2012 American Institute of Physics

As Published: http://dx.doi.org/10.1063/1.4771605

Publisher: American Institute of Physics (AIP)

Persistent URL: http://hdl.handle.net/1721.1/80729

Version: Final published version: final published article, as it appeared in a journal, conference proceedings, or other formally published context

Terms of Use: Article is made available in accordance with the publisher's policy and may be subject to US copyright law. Please refer to the publisher's site for terms of use. 


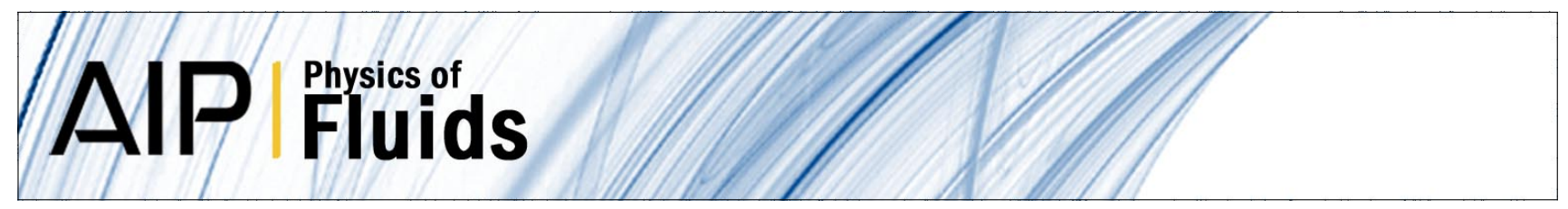

\section{Droplets bouncing on a wet, inclined surface}

Tristan Gilet and John W. M. Bush

Citation: Phys. Fluids 24, 122103 (2012); doi: 10.1063/1.4771605

View online: http://dx.doi.org/10.1063/1.4771605

View Table of Contents: http://pof.aip.org/resource/1/PHFLE6/v24/i12

Published by the American Institute of Physics.

\section{Related Articles}

Oscillations of a gas pocket on a liquid-covered solid surface

Phys. Fluids 24, 122101 (2012)

Dynamics of droplet coalescence in response to increasing hydrophobicity

Phys. Fluids 24, 112105 (2012)

A novel method to produce small droplets from large nozzles

Rev. Sci. Instrum. 83, 115105 (2012)

Thermal boundary layer analysis corresponding to droplet train impingement

Phys. Fluids 24, 112102 (2012)

Drop impact and wettability: From hydrophilic to superhydrophobic surfaces

Phys. Fluids 24, 102104 (2012)

\section{Additional information on Phys. Fluids}

Journal Homepage: http://pof.aip.org/

Journal Information: http://pof.aip.org/about/about_the_journal

Top downloads: http://pof.aip.org/features/most_downloaded

Information for Authors: http://pof.aip.org/authors

\section{ADVERTISEMENT}

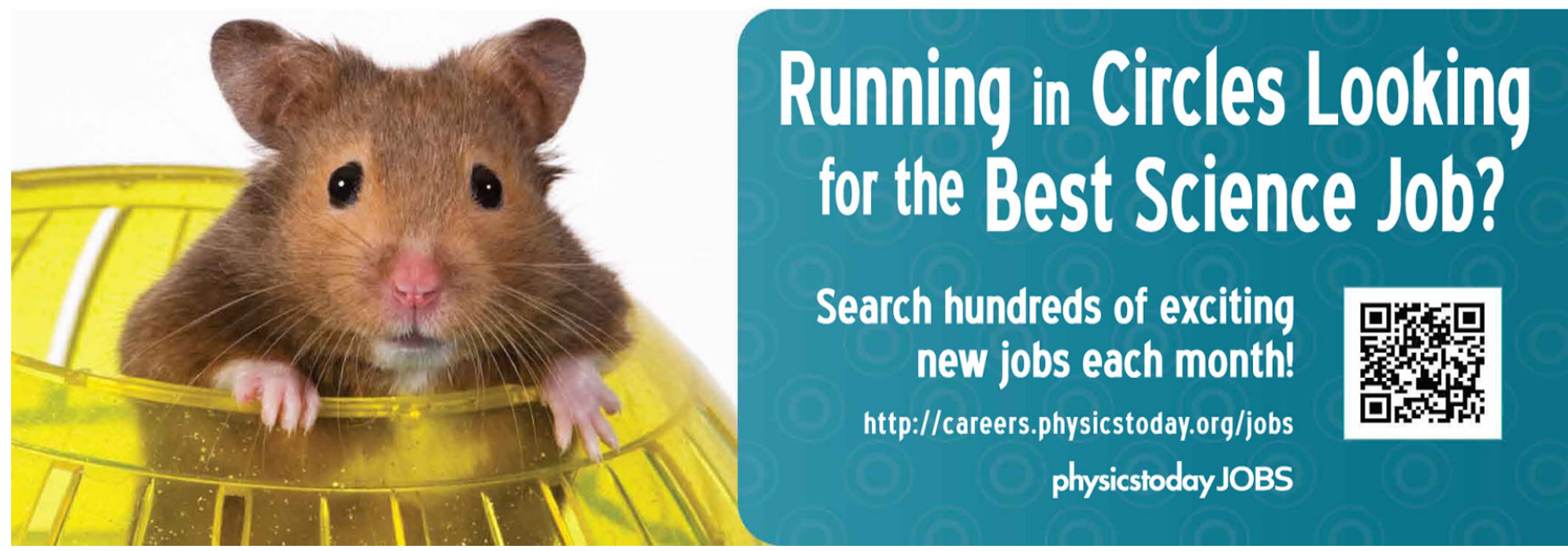




\title{
Droplets bouncing on a wet, inclined surface
}

\author{
Tristan Gilet and John W. M. Bush ${ }^{\text {a) }}$ \\ Department of Mathematics, Massachusetts Institute of Technology, \\ 77 Massachusetts Avenue, Cambridge, Massachusetts 02139
}

(Received 18 May 2012; accepted 6 November 2012; published online 13 December 2012)

\begin{abstract}
We present the results of an experimental investigation of fluid drops impacting an inclined rigid surface covered with a thin layer of high viscosity fluid. We deduce the conditions under which droplet bouncing, splitting, and merger arise. Particular attention is given to rationalizing the observed contact time and coefficients of restitution, the latter of which require a detailed consideration of the drop energetics. (c) 2012 American Institute of Physics. [http://dx.doi.org/10.1063/1.4771605]
\end{abstract}

\section{INTRODUCTION}

Drop impact is a crucial step in many natural and industrial processes. The impact of raindrops is responsible for the aeration of lakes ${ }^{1}$ and the propagation of many forms of life, ${ }^{2}$ including fungi and bacteria. ${ }^{3}$ Impact is also determinant for spray coating (with paints or plant treatments), ${ }^{4}$ fuel injection, ${ }^{5}$ surface cooling, ${ }^{6}$ and ink-jet printing. ${ }^{7}$ Considerable recent attention has been given to drop impact on superhydrophobic surfaces, with a view to rationalizing their self-cleaning abilities. ${ }^{8}$ On more fundamental grounds, droplets bouncing on a vertically vibrated liquid bath exhibit remarkable behaviors reminiscent of quantum particles ${ }^{9}$ including single-particle diffraction, ${ }^{10}$ quantized orbits, ${ }^{11}$ and tunneling. ${ }^{12}$

When a liquid drop impacts another liquid body, either bath, drop or film, it can either coalesce or bounce, remain in one piece or split into several droplets. Lord Rayleigh ${ }^{13}$ first reported such behavior in the interactions between two colliding streams of droplets. His work has since been extended in many directions. ${ }^{14-16}$ We first consider drop impact on a fluid bath. As a drop approaches the bath, air is expelled from the intervening air layer. The drop will bounce or coalesce according to whether this layer drains to a critical thickness during impact. While this critical thickness depends in general on both the fluid properties and system cleanliness, it is typically on the order of a few micrometers, according to the interference fringes visible when the drop is illuminated with a monochromatic source. ${ }^{17,18}$ If a sub-micrometric thickness is reached, the air layer ruptures in response to the cohesive Van Der Waals forces acting between the liquids. Then, the large curvature of the interface adjoining the merger point causes the air film between drop and bath to retract quickly, prompting drop coalescence. To bounce, this merger must be avoided through the sustenance of an air layer that exerts a lubrication force ${ }^{19}$ and so causes the drop to deform, decelerate, and ultimately reverse direction.

Bouncing objects are subject to universal mechanisms that depend only weakly on the particular configuration. Momentum should be reversed in a finite time by a restoring force, as in a spring. The translational kinetic energy is converted into deformational potential energy associated with surface tension for liquids, and elasticity for solids. This energy is then partly restored to the translational motion following impact. For the case of a bouncing drop, the remaining energy is fed into internal drop motion, including waves and oscillations, and the concomitant dissipation. The apparent contact time $t_{c}$ between the impacting object and the substrate is comparable in magnitude to the characteristic time of the energy transfer

$$
t_{c} \sim \sqrt{M / k}
$$

\footnotetext{
a)Electronic mail: bush@math.mit.edu.
} 
where $M$ is the mass of the bouncing object and $k$ the stiffness of the spring mechanism. For a droplet, this time corresponds to the capillary time $\tau_{\sigma}=\sqrt{M / \sigma}{ }^{20,21}$ Bouncing arises when the drainage time $t_{d}$ of the lubricating air layer is larger than $t_{c}$; otherwise coalescence occurs. Drainage time measurements are rarely reproducible, ${ }^{22}$ as $t_{d}$ depends not only on the properties (density, viscosity, surface tension, and rheology) of both fluids ${ }^{23,24}$ but on temperature, vapor concentration, solubility of the interstitial gas, electrostatic fields, and the presence of surfactants and dust. ${ }^{13,25,26}$

Coalescence may be significantly delayed by injecting air into the intervening layer to compensate for drainage. ${ }^{27}$ This may be achieved in various ways, including horizontal relative motion between the droplet and the bath ${ }^{28-31}$ and thermocapillary effects. ${ }^{29} \mathrm{~A}$ water droplet placed in the vicinity of a very hot source (a solid surface ${ }^{32,33}$ or a bath $^{34}$ ) is observed to float on a gas cushion, as first noted by Leidenfrost (1756). ${ }^{63}$ The air film transfers the heat to the bottom of the droplet which evaporates; the released vapor balances the losses due to drainage. Coalescence may also be avoided by vibrating the underlying interface. ${ }^{35-37}$ The droplet then bounces indefinitely like an inelastic ball, ${ }^{38}$ the energy dissipated by viscosity being balanced by the energy extracted from the vibration.

In the case of a droplet bouncing on a pool of liquid, the bouncing dynamics is particularly difficult to characterize, ${ }^{39}$ because both the droplet and the bath are subject to significant deformation and internal flow, these flows being coupled. A comprehensive model has been proposed for the relatively simple case of a droplet bouncing on a soap film, ${ }^{40}$ where only the droplet has a significant kinetic energy, and the soap film stores most of the surface energy. Another configuration of interest is a droplet bouncing on a rigid substrate. Several variants of this problem have recently been investigated: water droplets impacting superhydrophobic surfaces ${ }^{41}$ Leidenfrost droplets impacting a hot plate ${ }^{33}$ as well as the impact of liquid marbles (water drops coated with a hydrophobic powder $)^{42}$ and inflated balls. ${ }^{43}$ Among these configurations, the lubrication layer is maintained only in the Leidenfrost case: in the others, the bouncing object touches then detaches from the substrate. When drops strike a fluid bath or film, the maintenance of the intervening lubrication layer is facilitated by the smoothness of the fluid surfaces. Indeed, any roughness of the order of the air layer thickness would penetrate it and so initiate contact.

More recently, the bouncing of droplets on a thin layer of high viscosity fluid has been considered. ${ }^{36,44}$ This viscous layer ensures a smooth interface and so helps maintain the lubricating air film. The present paper represents a variant of this problem. Specifically, we investigate the impact of a droplet on an inclined solid surface coated with a thin layer of high viscosity fluid. The experimental setup is described in Sec. II. The impact is qualitatively discussed in Sec. III A. Quantitative measurements are presented in Sec. III B, and the energy transfers discussed in Sec. III C. Finally, the potential sources of energy dissipation are discussed in Sec. IV.

\section{EXPERIMENTAL SETUP}

Drops of silicon oil (Dow Corning - 1 to 100cS) impact a sheet of plexiglas coated with a submillimetric layer of silicon oil (Dow Corning - $100000 \mathrm{cS}$ ). The plexiglass makes an angle $\alpha \in\left[0^{\mathrm{O}}, 40^{\mathrm{O}}\right]$ with the horizontal. The incoming drops are released from a syringe; their volume corresponds to that of a sphere of radius $R=0.94 \pm 0.02 \mathrm{~mm}$. Experiments are recorded at 3000 fps with a high-speed camera (Photron FastCam SA5). The field of view and resolution are such that a pixel corresponds to $23 \mu \mathrm{m}$. Figure 1(a) shows an overview of a typical bouncing event, made by superposing successive frames. The initial drop (Fig. 1(b)) splits into several daughter droplets (Fig. 1(c)) that later also bounce on the surface. When the drop merges with the underlying oil layer, we shift the substrate so that the next drop impacts an undisturbed area. A series of such impact events are presented in Figure 2.

The relevant droplet variables are the radius $R$, viscosity $\nu$, density $\rho$, surface tension $\sigma$, the impacting velocity $V$, and gravity $g$. Based on these variables, three dimensionless numbers can be formed: the Bond number $B o=\rho g R^{2} / \sigma$, the Weber number $W e=\rho R V^{2} / \sigma$, and the Ohnesorge number $O h=v \sqrt{\rho} / \sqrt{\sigma R}$. Bo and We prescribe the relative importance of gravity and inertia to surface tension, respectively, while $O h$ indicates the relative magnitudes of the capillary time $\tau_{\sigma}=\sqrt{\rho R^{3} / \sigma}$ and viscous time $\tau_{v}=R^{2} / \nu$. The Weber number is used to characterize the impact. For inclined impacts, we make the distinction between the normal Weber number $W e_{1 n}=\rho R V_{1 n}^{2} / \sigma$ 


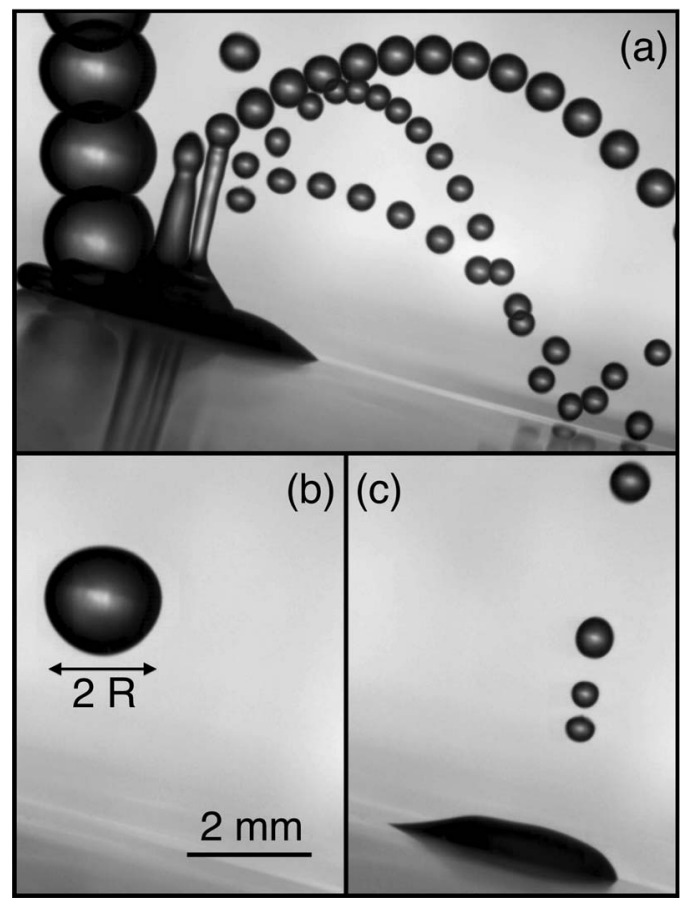

FIG. 1. A drop impacts an inclined coated surface and splits into four droplets. (a) Superposition of successive frames, taken at $300 \mathrm{fps}$, reveals the trajectory of the droplets. (b) The initial drop, before impact (time $t=-7 \mathrm{~ms}$ ). (c) The satellite droplets formed after impact (time $t=16 \mathrm{~ms}$ ). Dimensionless parameters are $W e_{1 n}=8.26, \mathrm{Oh}=7 \times 10^{-3}, \mathrm{Bo}=0.4$ and $\alpha=14^{\circ}$.
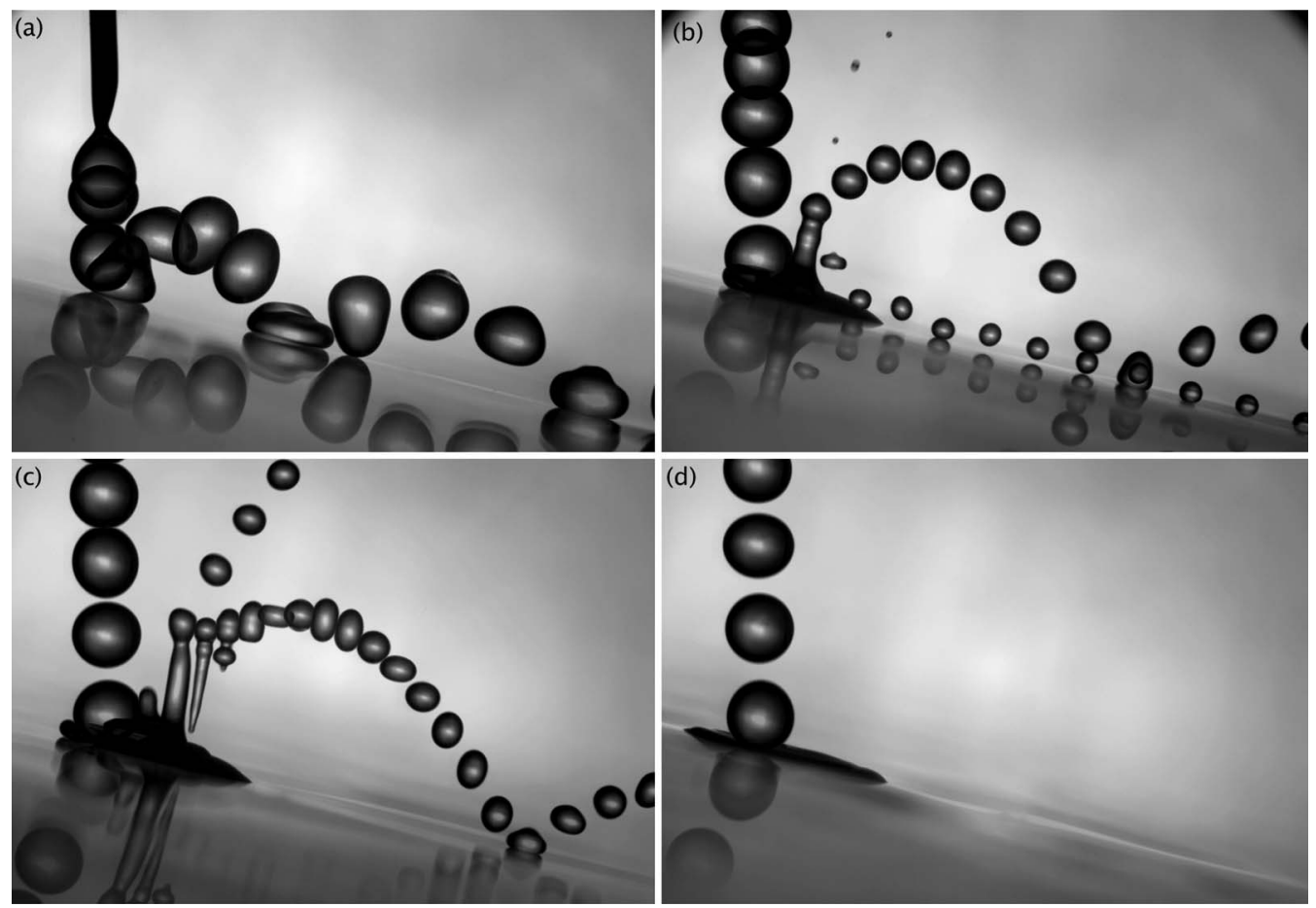

FIG. 2. Impact scenarios at different Weber number $W e_{1 n}$, as revealed through a superposition of successive frames separated by $\delta t$ milliseconds. (a) $W e_{1 n}=1.7, \delta t=10 \mathrm{~ms}$. (b) $W e_{1 n}=5.8, \delta t=6 \mathrm{~ms}$. (c) $W e_{1 n}=12.1, \delta t=4 \mathrm{~ms}$. (d) $W e_{1 n}=15.3$, $\delta t=4 \mathrm{~ms}$. Other dimensionless parameters are $O h=7 \times 10^{-3}, B o=0.4$ and $\alpha=14^{\circ}$. A detailed analysis of these impacts is found in Fig. 4. 


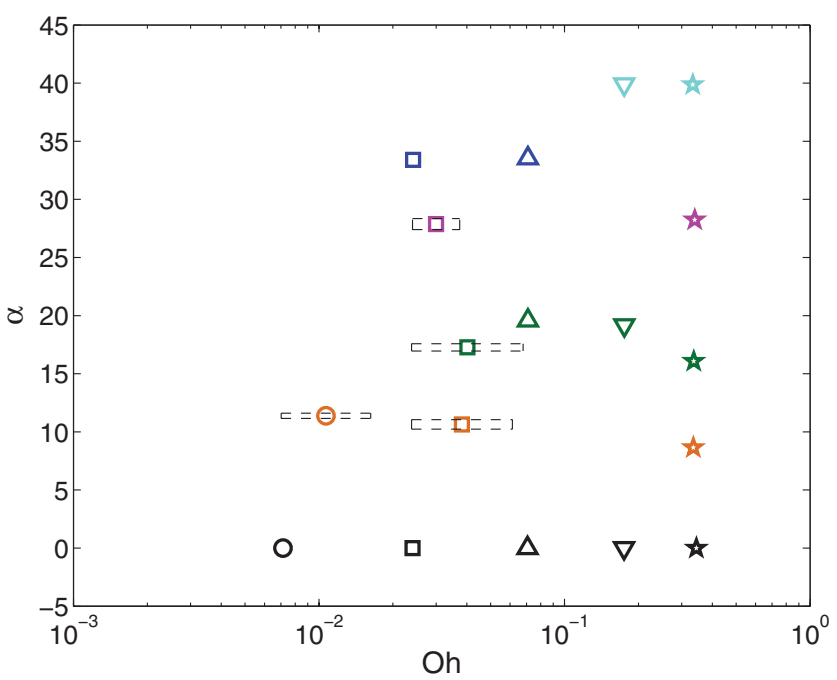

FIG. 3. Shade code used in our presentation of experimental results. Different symbols correspond to different Ohnesorge numbers $O h$ (between 0.007 and 0.35 ), while the shade indicates the value of the inclination angle $\alpha$. Experiments with slightly different values of $(O h, \alpha)$ are grouped together with the same symbol; the dashed rectangle indicates the region described by the symbol. In subsequent figures, $B o=0.42 \pm 0.04$ is denoted by bold symbols. The satellite droplets have a smaller $B o$, and so are represented by plain symbols.

and the tangential Weber number $W e_{1 t}=\rho R V_{1 t}^{2} / \sigma$, where $V_{1 n}$ and $V_{1 t}$ are the impact velocity normal and tangent to the surface, respectively. The Bond number, the Ohnesorge number, and the angle $\alpha$ are then represented by the shade code defined in Fig. 3 .

\section{EXPERIMENTAL RESULTS}

\section{A. Phenomenology}

Figure 4 shows sequences of six identical droplets impacting an inclined surface. The parameters are the same in all experiments $\left(\alpha=14^{\circ}, O h=0.007\right.$, and $\left.B o=0.4\right)$, except for the Weber number, which is varied between 1.7 and 15.3. At impact $(t=0)$, a set of capillary waves rises from the base of the drop to form a pyramidal structure $\left(t=0.13 \tau_{\sigma}\right)$. These large amplitude waves then reshape the droplet into a pancake $\left(t=0.40 \tau_{\sigma}\right)$. This phase is identical for all six sequences, and qualitatively similar to the impact of water droplets on superhydrophobic surfaces. ${ }^{45,46}$ What happens next is more specific to impact on thin films, and depends strongly on the incident normal Weber number $W e_{1 n}$.

At $W e_{1 n}<2$ [Fig. 4(a)], the air layer survives the impacting droplet, so there is no merger. The droplet strongly deforms, then takes off and recovers its spherical shape. This regime is called complete bouncing and will be further detailed in Secs. III B and III C. When $W e_{1 n}>15$ [Fig. 4(f)], the air layer breaks and total merger occurs between the droplet and the underlying film. As $W e_{1 n}$ is increased progressively between these two extremes, a relatively complex series of intermediate regimes is found [Figs. 4(b)-4(e)], where the incoming drop splits into a number of outgoing daughter droplets.

First, for $W e_{1 n}>2$, the pancake formed by the capillary waves at $t \simeq 0.4 \tau_{\sigma}$ evolves into a torus. The subsequent collapse of this torus is accompanied by the emission of a Worthington jet $\left(t \simeq 0.6 \tau_{\sigma}\right)$ that pinches off into several tiny satellite droplets at $t \simeq 0.7 \tau_{\sigma}$ when $W e_{1 n}<7.5$ [Figs. 4(b) and 4(c)]. The volume of these droplets is shown in Fig. 5 (medium gray circles-red). The air layer survives impact when $W e_{1 n}<4.1$ [Figs. 4(a) and 4(b)]. For larger $W e_{1 n}$, it breaks at a time $t \simeq 0.6 \tau_{\sigma}$ after impact, and a significant amount of the initial drop (at least $70 \%$ in volume) merges with the underlying film [Figs. 4(c)-4(f)]. 


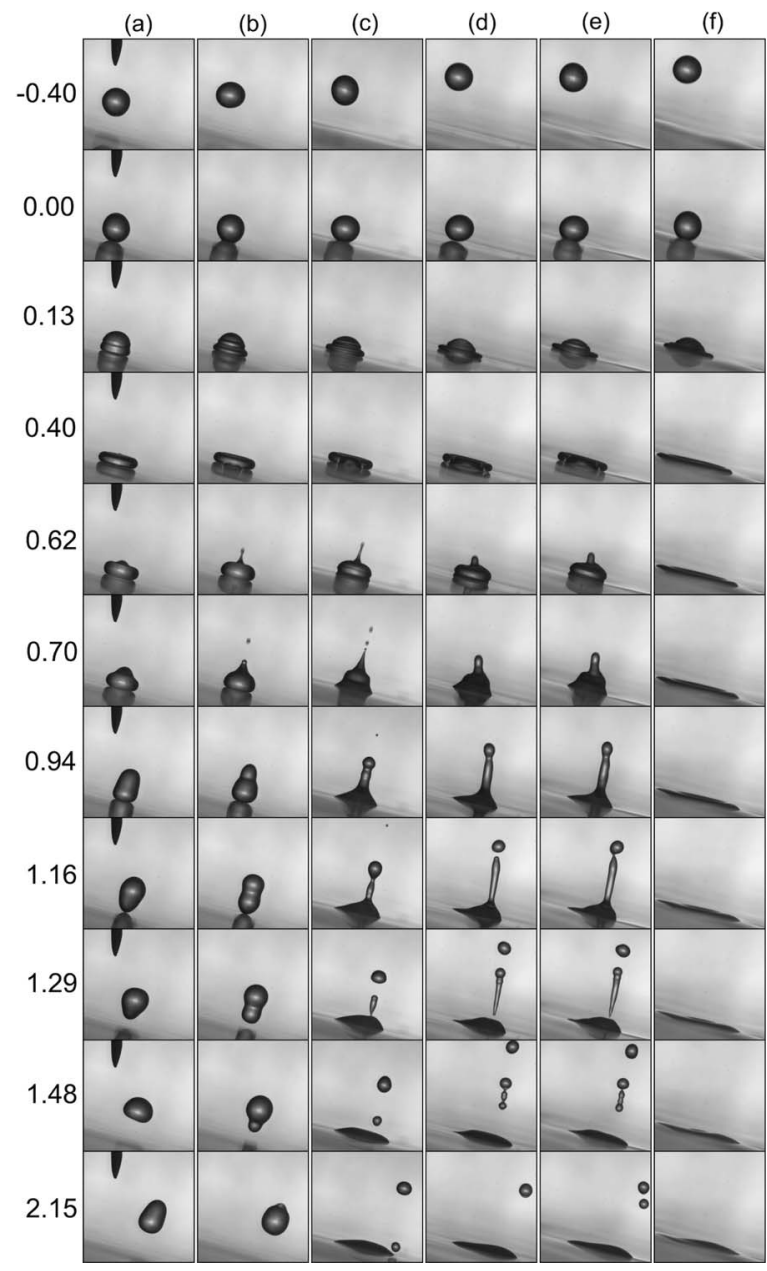

FIG. 4. Bouncing, splitting, and merging: (a) $W e_{1 n}=1.7$, (b) $W e_{1 n}=3.9$, (c) $W e_{1 n}=5.8$, (d) and (e) $W e_{1 n}=12.1$, (f) $W e_{1 n}=15.3$. The other parameters are fixed for each droplet impact $\left(\alpha=14^{\circ}, O h=0.007\right.$ and $\left.B o=0.4\right)$. The frames are taken at identical times after impact, normalized by the capillary time $\tau_{\sigma}=12.4 \mathrm{~ms}$ and indicated in the left column.

The merger also generates nonlinear capillary waves that converge and strongly distort the top of the drop for $t \geq 0.9 \tau_{\sigma}$. The resulting extension is usually followed by two pinching events: one close to the top $\left(t \simeq 1.2 \tau_{\sigma}\right)$, then one at the bottom $\left(t \simeq 1.3 \tau_{\sigma}\right)$. The liquid filament in between quickly retracts, but may still pinch off into several satellite droplets. Their volume is reported in Fig. 5 (light gray circles-green). The retraction is especially sensitive to initial conditions, and two drops impacting with almost identical $W e_{1 n} \simeq 12.1$ ultimately split into a different number of satellite droplets [Figs. 4(d) and 4(e)].

For larger values of $\mathrm{Oh}$, the transition to merger is observed at higher $\mathrm{We}_{1 n}$ (typically around 15), indicating that the dynamics of the air layer strongly depends on droplet viscosity (as suggested in Ref. 36). Moreover, the capillary waves are damped by viscosity and the drop does not experience sufficient deformation to split. Pinching is only observed for $O h<0.03$, the same criterion as for a quiescent droplet coalescing with an underlying liquid bath. ${ }^{47,48}$

\section{B. Bouncing as a black box}

In the complete bouncing events, the contact time $t_{c}$ is measured as the time when the droplet is in apparent contact with the substrate. For bouncing droplets, regardless of the configuration, $t_{c}$ always scales with the capillary time $\tau_{\sigma} \cdot{ }^{41}$ Indeed, the capillary time corresponds to the characteristic 


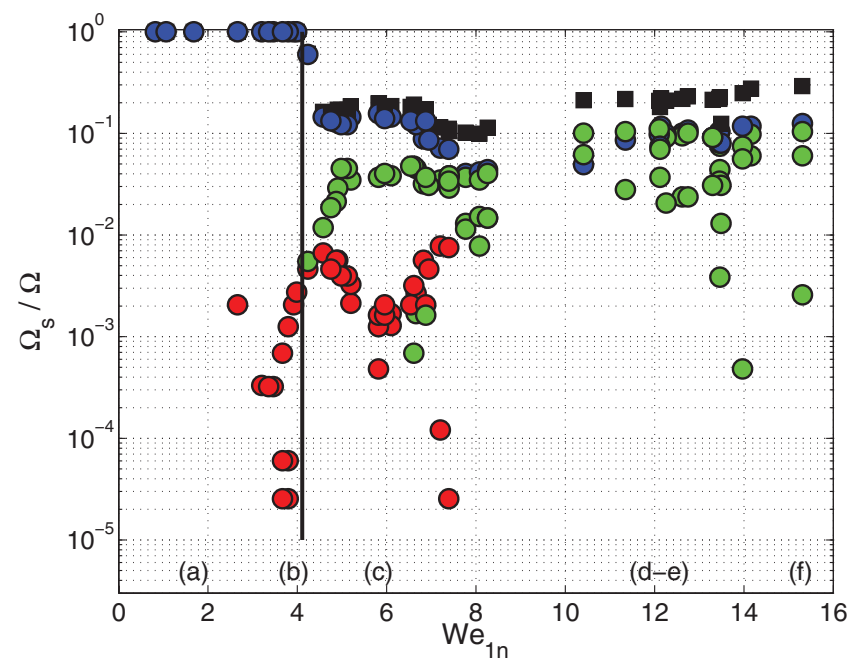

FIG. 5. Volume of the satellite droplets $\Omega_{s}$ normalized by the volume of the impacting drop $\Omega$, as a function of the incident normal Weber number $W e_{1 n}$. Other parameters are $O h=0.007, B o=0.4$ and $\alpha=14^{\circ}$. (Blue circle) Main droplet. (Red circle) Worthington satellite droplets, ejected above the main drop at $t \simeq 0.7 \tau_{\sigma}$. (Green circle) Satellite droplets from the pinch off, ejected below the main drop at $t \in[1.16,1.48] \tau_{\sigma}$. (Black square) Total volume ejected after impact.

frequency at which kinetic and surface energy can be exchanged. The normalized contact time $t_{c} / \tau_{\sigma}$ is reported in Fig. 6 as a function of $W e_{1 n}$. The slight decrease of $t_{c} / \tau_{\sigma}$ with increasing $W e_{1 n}$ has also been observed and rationalized in other bouncing configurations, including water droplets impacting soap films ${ }^{40}$ and superhydrophobic surfaces. ${ }^{21}$

The bouncing event can be crudely seen as a black box that modifies the trajectory of the center-of-mass of the droplet (Fig. 7). The input and output states are defined as the points where this drop's center-of-mass is a perpendicular distance $R$ from the surface. The incoming droplet is usually spherical, so the input state is clearly defined. Conversely, the outgoing droplet is elongated, so the output time may occur before lift-off. In this case, the trajectory after lift-off must be extrapolated backwards in order to define the virtual output state. The time delay $\Delta t$ between the input and the

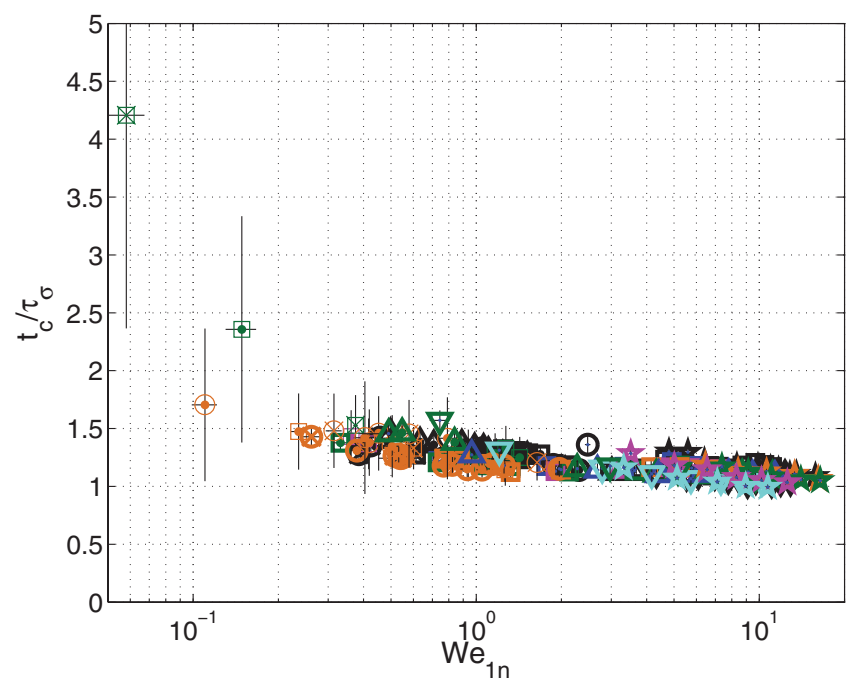

FIG. 6. Normalized contact time $t_{c} / \tau_{\sigma}$ as a function of the incident normal Weber number $W e_{1 n}$ in the case of complete bouncing. Symbols are defined in Figure 3. 


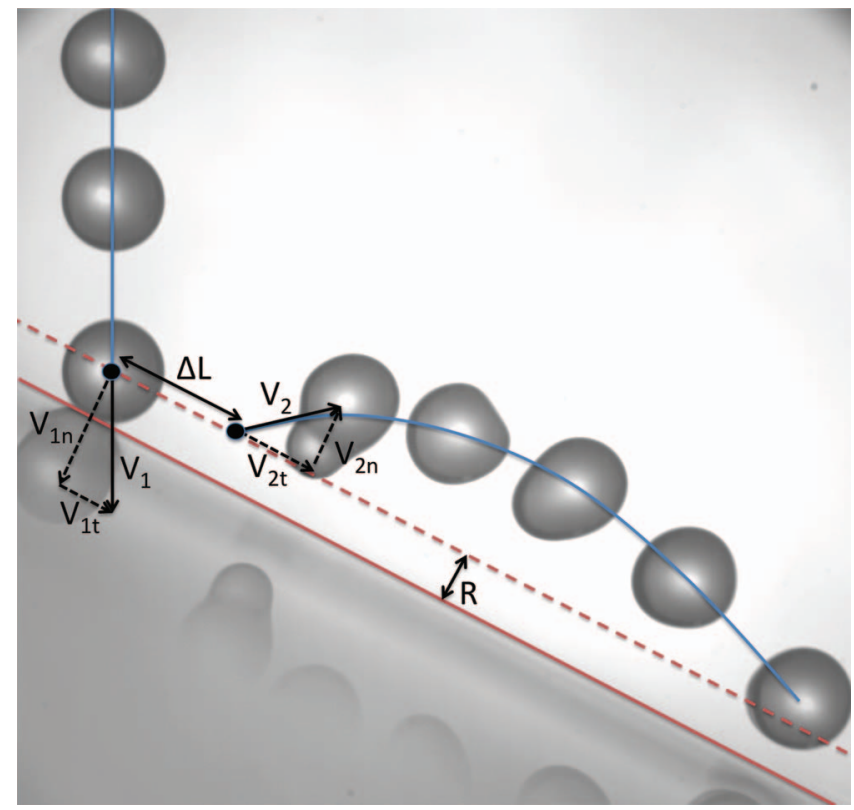

FIG. 7. Bouncing is seen as a black box that modifies the trajectory of the center-of-mass of the droplet, changing the velocity from $\vec{V}_{1}$ to $\vec{V}_{2}$.

output, normalized by the contact time, is represented in Fig. 8 as a function of $W e_{1 n}$; the ratio is always less than 1 , indicating that the droplet is prolate when it lifts off.

The slip length $\Delta L$ is defined as the distance between the input and the output states. It can be estimated by considering that the droplet slides without friction, so the distance traveled should be

$$
\Delta L_{0}=V_{1 t} \Delta t+g \sin \alpha \frac{\Delta t^{2}}{2} .
$$

Figure 9 confirms this prediction, showing that $\Delta L / \Delta L_{0}$ is very close to 1 . The slight decrease observed for large $W e_{1 n}$ indicates that a droplet squeezed by a relatively violent impact experiences

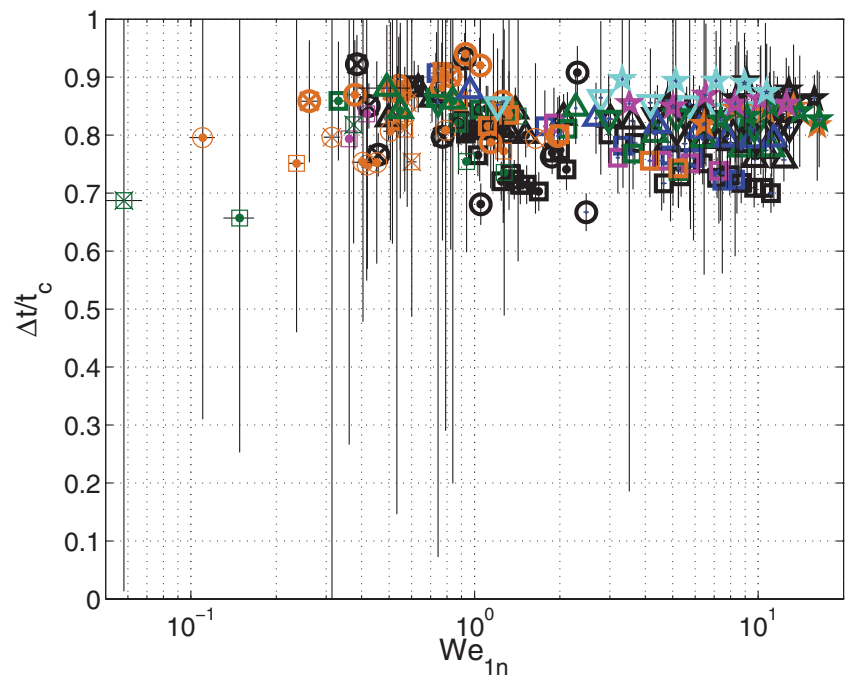

FIG. 8. Normalized time delay $\Delta t / t_{c}$ as a function of the incident normal Weber number $W e_{1 n}$. Symbols are defined in Figure 3 . 


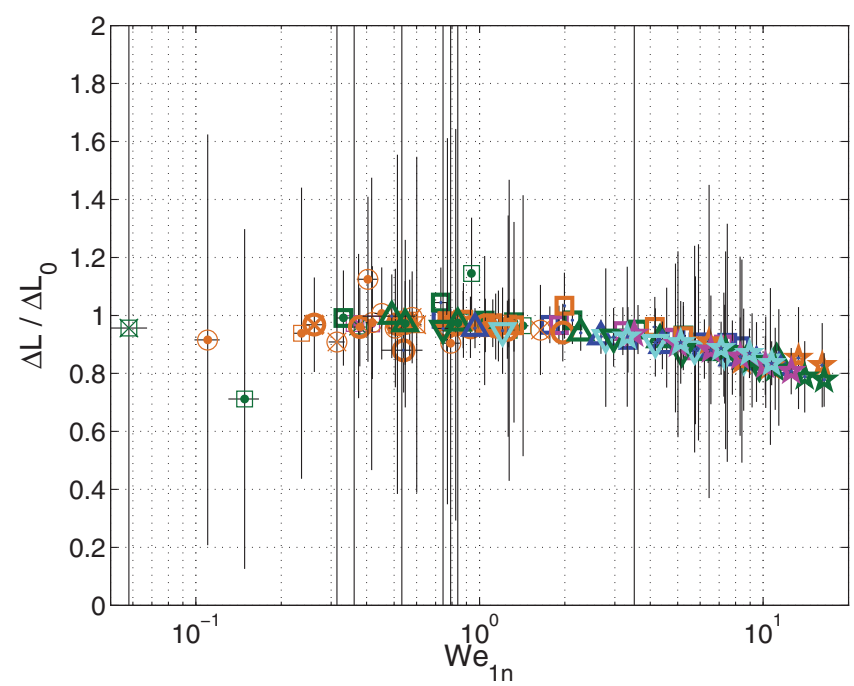

FIG. 9. Slip length $\Delta L$, normalized by the prediction $\Delta L_{0}$ [Eq. (2)], as a function of the incident normal Weber number $W e_{1 n}$. Symbols are defined in Figure 3.

an increased friction from the lubricating air layer. The normalized slip length depends on neither Oh nor $\alpha$.

The change of normal velocity is quantified through the ratio of the normal Weber number after and before impact, which corresponds to the square of the normal coefficient of restitution $\epsilon_{n}=V_{2 n} / V_{1 n} . \epsilon_{n}$ does not depend on $\alpha$ (Fig. 10), as was noted by Anders et al. ${ }^{49}$ moreover, it is observed that

$$
\frac{W e_{2 n}}{W e_{1 n}}=\epsilon_{n}^{2}= \begin{cases}F(\mathrm{Oh}) & \text { when } W e_{1 n}<1 \\ F(\mathrm{Oh}) \cdot W e_{1 n}^{-1 / 2} & \text { when } W e_{1 n}>1 .\end{cases}
$$

A similar decrease of $\epsilon_{n}$ with increasing $W e_{1 n}$ has been reported for inflated balls, ${ }^{43}$ drops impacting a deep bath, ${ }^{39}$ drops impacting superhydrophobic surfaces, ${ }^{41}$ Leidenfrost drops,${ }^{33}$ and liquid marbles ${ }^{42}$ Solid balls do not experience this decreased bouncing efficiency with increasing kinetic energy, ${ }^{50-53}$

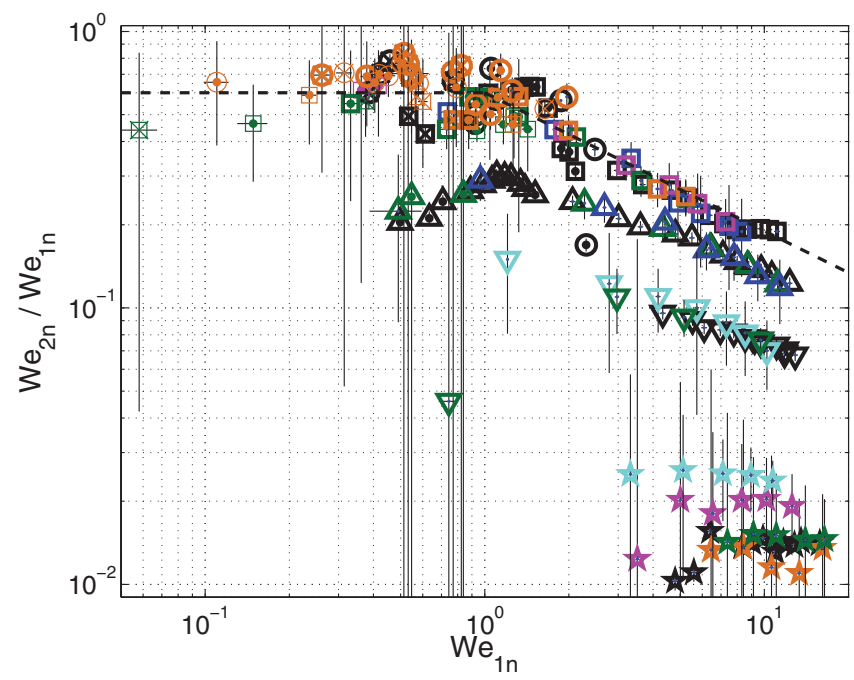

FIG. 10. Normal coefficient of restitution: Ratio of the normal Weber number after and before impact, as a function of $W e_{1 n}$. The dashed curve corresponds to Eq. (3) with $F(\mathrm{Oh})=0.6$. Symbols are defined in Figure 3. 


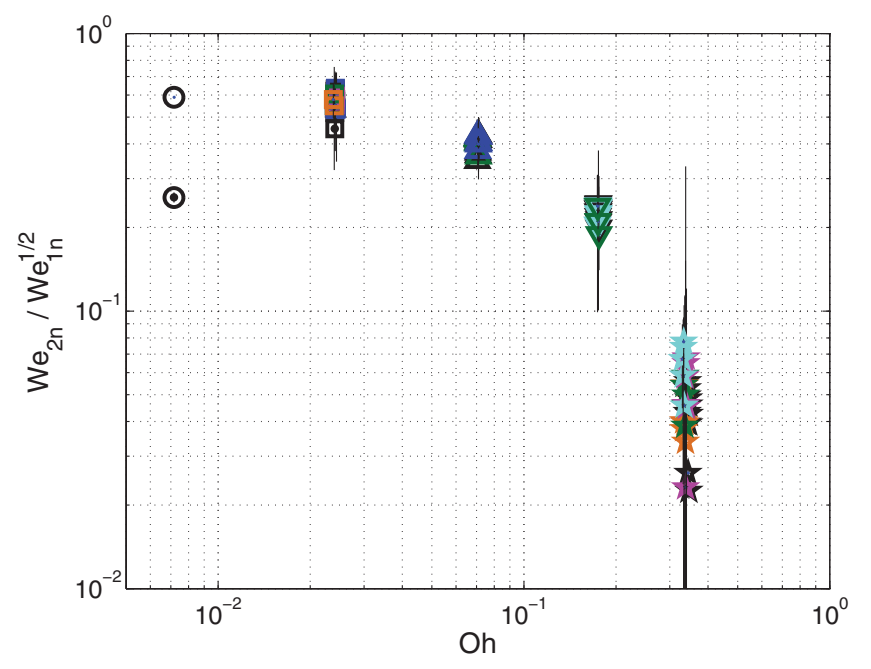

FIG. 11. Prefactor $F(O h)$ as defined in the scaling law (3). Symbols are defined in Figure 3.

which suggests that this trend is mainly due to the deformation experienced by impacting liquid objects. Nevertheless, the scaling behavior associated with this decrease is not universal; for example, for Leidenfrost droplets, ${ }^{33} \epsilon_{n}>1$ for $W e_{1 n}<1$ and $\epsilon_{n}^{2} \sim W e_{1 n}^{-1}$. We suspect that this difference is due to vapor thrust in the Leidenfrost system, a physical effect absent in our configuration (cf. the Appendix).

The prefactor $F(O h)$ is approximately constant and equal to 0.6 for $O h<0.04$, then decreases sharply for larger $\mathrm{Oh}$ (Fig. 11). The normal coefficient of restitution drops to zero, indicating that the energy is completely dissipated by viscous effects during impact. In other words, the time taken by momentum to diffuse over the droplet $R^{2} / v$ is comparable to the contact time $t_{c}$, which prevents an efficient storage and recovery of the kinetic energy of the impacting drop. Note that at large $O h$, the scaling law (3) is no longer strictly observed, hence the apparent scatter in $F(O h)$.

The ratio of the tangential Weber number after and before impact, corresponding to the square of the tangential coefficient of restitution $\epsilon_{t}=V_{2 t} / V_{1 t}$, is represented in Fig. 12 as a function of $W e_{1 n}$. The absence of collapse and the presence of values higher than unity are due to the fact that

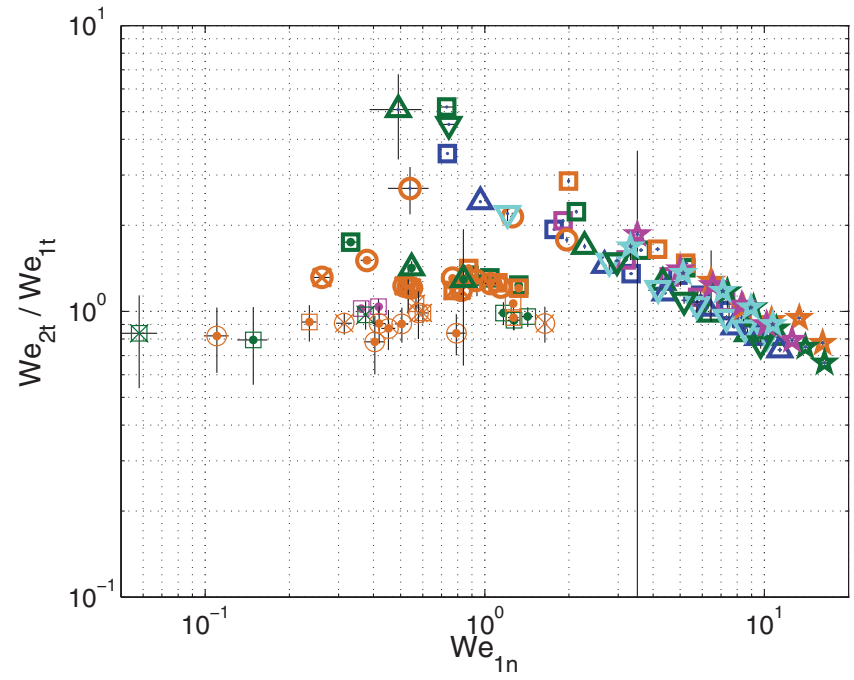

FIG. 12. Tangential coefficient of restitution: ratio of the tangential Weber number after and before impact, as a function of $W e_{1 n}$. Symbols are defined in Figure 3. 


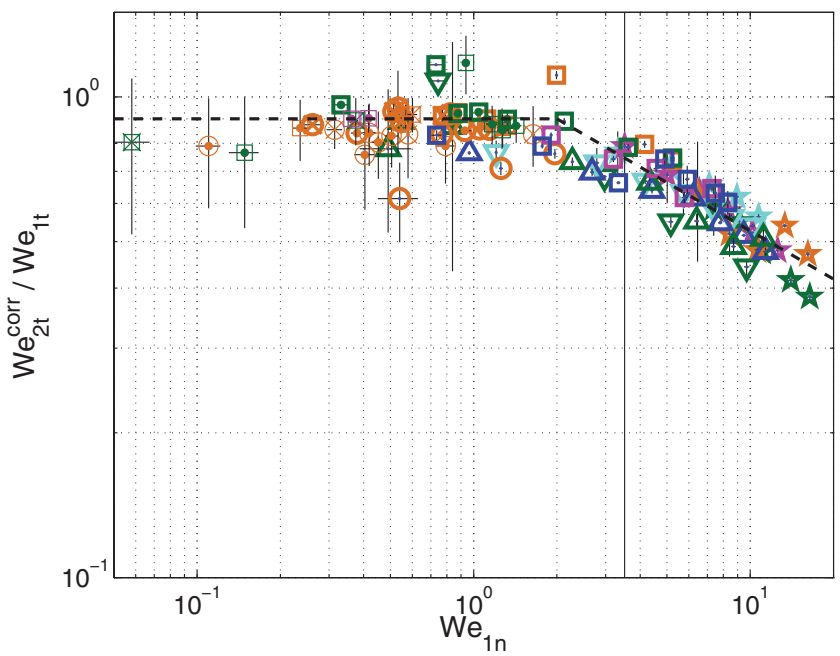

FIG. 13. Corrected tangential coefficient of restitution $W e_{2 t}^{c o r r}$ [defined in Eq. (4)], as a function of $W e_{1 n}$. The dashed line corresponds to Eq. (5). Symbols are defined in Figure 3.

gravity accelerates the droplet during the sliding contact. We can correct for this effect by defining a new tangential Weber number after impact, based on the final tangential velocity $V_{2 t}$

$$
\begin{aligned}
& V_{2 t, \text { corr }}=V_{2 t}-g \Delta t \sin \alpha, \\
& W_{2 t}^{\text {corr }}=\frac{\rho R V_{2 t, \text { corr }}^{2} .}{\sigma} .
\end{aligned}
$$

A much better collapse is observed with this correction (Fig. 13). The tangential coefficient of restitution then obeys a similar scaling law to the normal coefficient of restitution

$$
\frac{W e_{2 t}^{c o r r}}{W e_{1 t}}=\epsilon_{t}^{2}=\left\{\begin{array}{ll}
0.9 & \text { when } W e_{1 n}<2 \\
0.9\left(W e_{1 n} / 2\right)^{-1 / 3} & \text { when } W e_{1 n}<2
\end{array} .\right.
$$

It is noteworthy that the forms of the tangential (5) and normal (6) coefficients of restitution are quite different. In Eq. (5), the prefactor no longer depends on the Ohnesorge number, indicating that the tangential coefficient of restitution is independent of drop viscosity. In fact, $\epsilon_{t}$ seems to depend only on $W e_{1 n}$. The exponent of the scaling law is also different from that on $\epsilon_{n}$. Tangential coefficients of restitution are rarely reported in the droplet literature, ${ }^{54}$ and to our knowledge, no other data are available for comparison.

We note that the decomposition into normal and tangential coefficients of restitution suggests the long term behavior of droplets bouncing repeatedly on an inclined planar surface. The normal velocity monotonically decreases from one rebound to the next. Conversely, the decrease in tangential velocity due to energy dissipation may be balanced by the acceleration of gravity. Thus, provided the impact does not initiate merger, we expect that droplets will always end up sliding (and possibly rolling) on the surface. The dynamics of such sliding states is beyond the scope of this study.

\section{Energy transfers and the normal coefficient of restitution}

Both coefficients of restitution decrease significantly with increasing $W e_{1 n}$ in many bouncing droplet configurations. In this section, we attempt to rationalize the dependency of $\epsilon_{n}$ on $W e_{1 n}$ for low $\mathrm{Oh}$, by focusing on the normal impact on a horizontal surface. The translational energy (kinetic and gravity) and the surface energy of the droplet are measured for each frame by image processing. Surface energy is not easy to estimate when the shape becomes toroidal. Here, we calculate the surface of the convex envelope (i.e., the pancake shape). This yields a systematic error in the surface energy once the torus forms. Nevertheless, for a torus of large radius $R$ and small radius $a$, the surface 


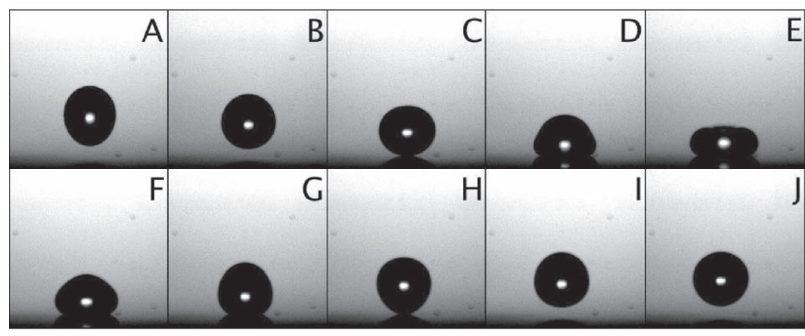

(a)

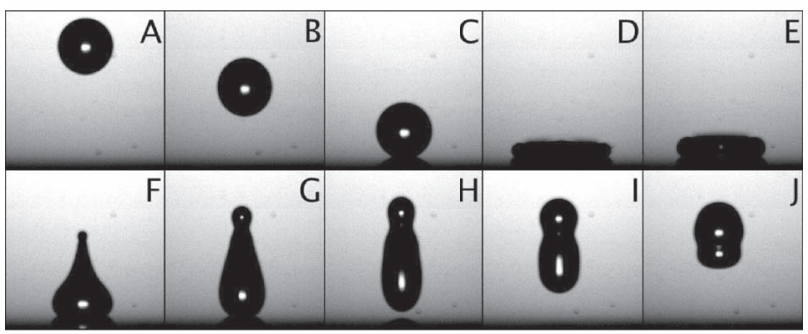

(c)
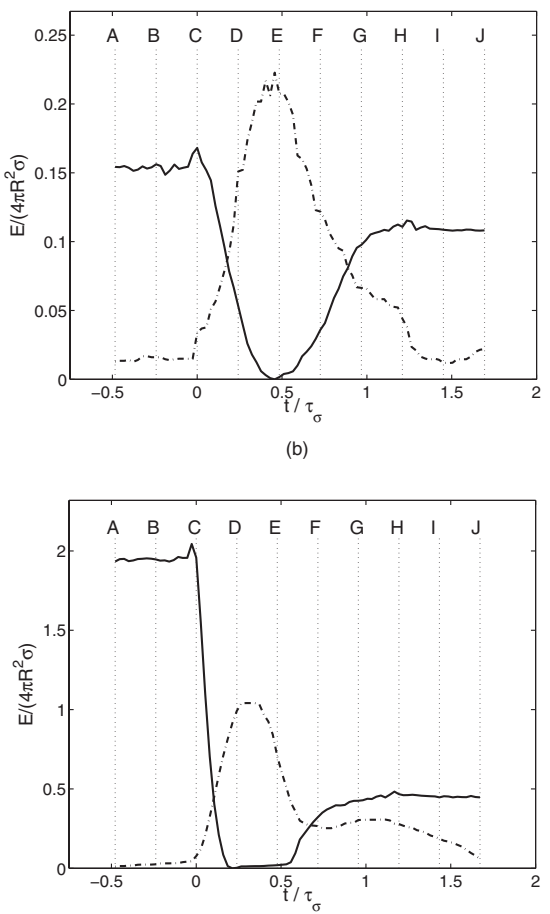

(d)

FIG. 14. Time evolution of the energy, for $O h=0.02$ : (a) and (b) $W e=0.53$ and (c) and (d) $W e=11.1$. In (a) and (c), the snapshots are taken every $3 \mathrm{~ms}$, the third frame corresponding to the impact time $(t=0)$. In (b) and (d), the dashed and solid lines represent the surface energy of the droplet and the mechanical energy (kinetic + gravity) of its center-of-mass.

is $S_{0}=4 \pi^{2} R a$ while the surface of its convex envelope is $S_{1}=2 \pi R^{2}+2 \pi^{2} R a+4 \pi a^{2}$. The relative error is then

$$
\frac{S_{1}-S_{0}}{S_{0}}=\frac{1}{2 \pi} \frac{R}{a}-\frac{1}{2}+\frac{1}{\pi} \frac{a}{R}
$$

which is always less than $8.4 \%$ provided $R / a<3$. In Figs. 14(b) and 14(d), the time evolution of these quantities is graphed for two typical experiments, at low and high We (Figs. 14(a) and 14(c), respectively). In the first case (low We, Figs. 14(a) and 14(b)), the entire kinetic energy is stored in surface energy; then, about 55\% is converted back into translation energy. In the second case (high We, Figs. 14(c) and 14(d)), only about 55\% of the kinetic energy is converted into surface energy, then $45 \%$ of it restored to kinetic energy. This suggests that the additional energy loss responsible for the decrease in $\epsilon_{n}$ at high We mainly happens during the compression phase, specifically during the transfer of translational to surface energy. This is confirmed by observing that the stored energy $E_{S}$ (normalized by $4 \pi R^{2} \sigma$ ) scales as $W e_{1 n}^{1 / 2}$ for $W e_{1 n}>1$ (Fig. 15), while $W e_{2 n}$ is proportional to $E_{S}$ (Fig. 16).

We can thus envisage the following scenario. The translation energy restored after impact is proportional to whatever is stored in surface energy. The energy that can be stored is proportional to the work $W$ done by the lubrication force $F_{L}$ (exerted by the air layer) to slow down and compress the droplet. The lubrication force should scale as $F_{L} \sim m V_{1 n} / \tau_{\sigma}$, namely, the change of momentum $m V_{1 n}$ divided by the time of this change. In the regime considered, the time required for the drop to achieve its maximum deformation is proportional to the capillary time $\tau_{\sigma}$ and independent of $W e_{1 n}$ (Fig. 4), as was the case for Leidenfrost impacts. ${ }^{55}$ The work done is then $W=F_{L} \cdot \delta$, where $\delta$ is the vertical distance traveled by the center-of-mass of the droplet. For a low impact velocity, it is reasonable to assume that most of the kinetic energy is transferred into surface energy, and that the droplet shape is close to ellipsoidal. Thus,

$$
\sigma \delta^{2} \sim m V_{1 n}^{2}
$$




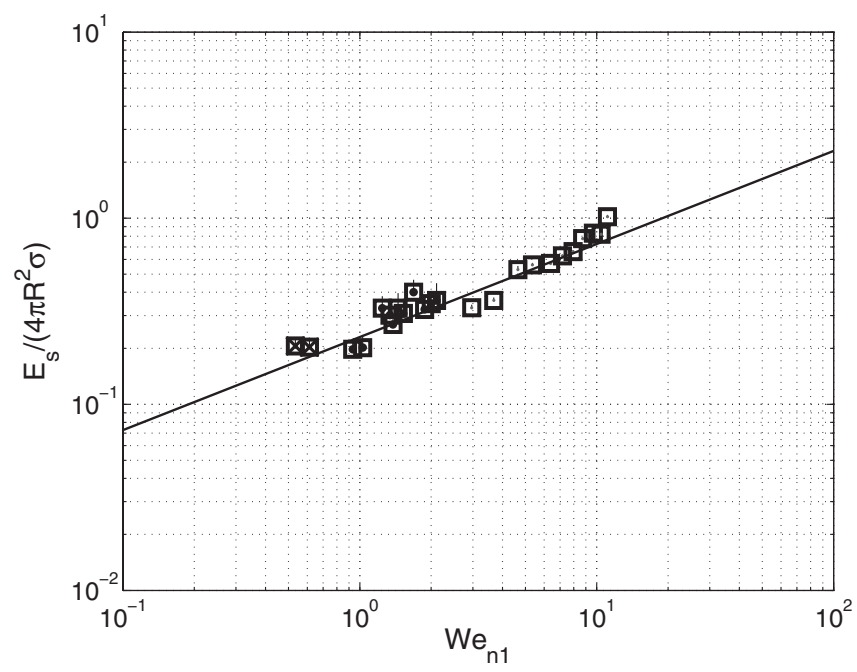

FIG. 15. Stored energy $E_{S}$, normalized by $4 \pi R^{2} \sigma$, as a function of the input Weber number $W e_{1 n}$, for $O h=0.02$. The solid line corresponds to the scaling law $E_{S} \sim W e_{1 n}^{1 / 2}$. Error bars are smaller than the symbol size.

so $W \sim W e_{1 n}$, and $\epsilon_{n}$ is constant (as observed at low $W e_{1 n}$ ). Nevertheless, the compression $\delta$ is limited by the radius of the droplet, and according to Eq. (7), $\delta \simeq R$ corresponds to $W e_{1 n} \sim 1$, the critical value of the transition between the two regimes in Fig. 10. When $W e_{1 n}>1, \delta \sim R$ so $W \sim m V_{1 n} R / \tau_{\sigma} \sim W e_{1 n}^{1 / 2}$. This scaling is consistent with the observation reported by Clanet et al. ${ }^{56}$ that the maximum spreading radius $R_{M}$ of an impacting drop scales as $W e_{1 n}^{1 / 4}$, so the corresponding surface energy scales as $\sigma R_{M}^{2} \sim W e_{1 n}^{1 / 2}$. Consequently, when $W e_{1 n}>1, \epsilon_{n} \sim W e_{1 n}^{-1 / 2}$ as observed experimentally.

\section{DISCUSSION}

Our experiments indicate that the kinetic energy available to the droplet when it impacts a thin liquid layer on a solid surface is partially, but not completely, transferred into surface energy. At high

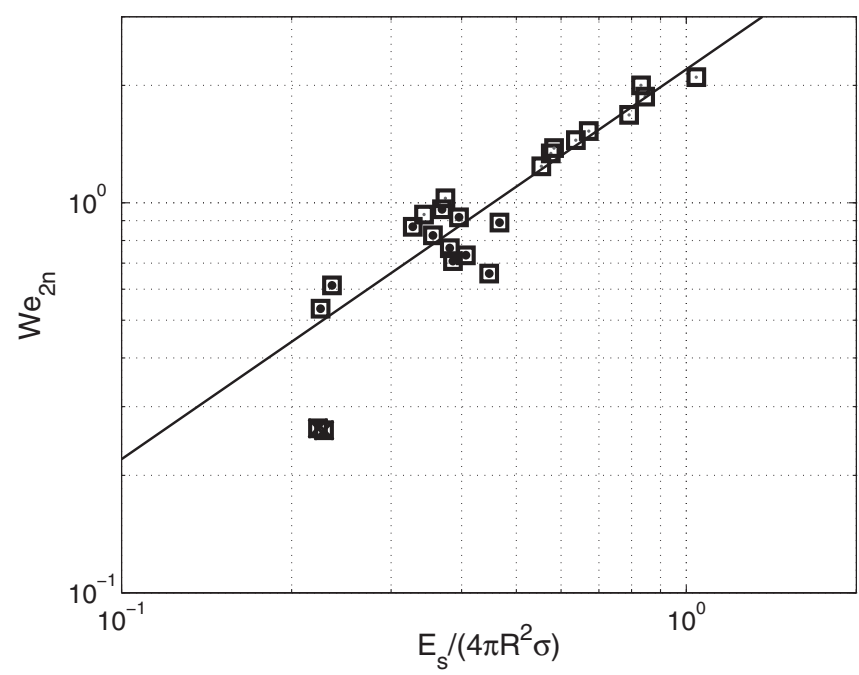

FIG. 16. Output Weber number $W e_{2 n}$ as a function of the stored energy $E_{S}$ normalized by $4 \pi R^{2} \sigma$. The solid line corresponds to the scaling law $W e_{2 n} \sim E_{S}$. Error bars are smaller than the symbol size. 
impact velocity, a significant part of this translational kinetic energy must be fed into internal flows. As direct viscous dissipation cannot occur on the short timescale of a rebound $(O h \ll 1$ indicates that the viscous time is much larger than the capillary time), these internal flows should persist, even when the droplet reaches its maximal deformation. However, it remains unclear what form these flows take. A PIV study of the droplet was deemed too challenging, due to the difficulties associated with the small scale of the system and the refraction of light at the droplet interface.

One potential mechanism for pumping the energy out and feeding internal flows would be the stretching of a vortex ring. The idea is based on Kelvin's theorem that states that for an inviscid flow, the circulation $\Gamma$ is conserved on a closed curve advected by the flow. Such a curve could be, for example, the contour of half the droplet cross section. In impacts on a solid surface, Kelvin's theorem is not strictly valid because of the presence of a boundary layer close to the surface, ${ }^{57}$ inside which circulation is created. In our experiment, however, this shear generation is at least partially mitigated by the presence of the lubricating air layer, and it is reasonable to assume that circulation is conserved when $O h \ll 1$. Suppose that, directly after impact, some circulation $\Gamma$ is present in the droplet, so that the associated vorticity scales as $\omega \sim \Gamma / R^{2}$. When the droplet flattens to a thickness $a$, the vorticity increases correspondingly $\omega \sim \Gamma / a^{2}$. The horizontal extension $L$ of the drop satisfies $L \sim \sqrt{R^{3} / a}$. The kinetic energy of the vortical flow then scales as $K_{\omega} \sim \rho a^{4} L \omega^{2} \sim \rho L \Gamma^{2}$. In a typical experiment at high We (e.g., Fig. 14(c)), the horizontal extension of the droplet doubles at most, so the kinetic energy of the vortex likewise doubles. For this energy to correspond to the loss observed in Fig. 14(d), the initial energy of the vortex would have to be about $50 \%$ of the total kinetic energy. This is substantially more than we should expect from the residual vorticity created when the droplet detaches from the nozzle. We conclude that vortex stretching is unlikely to be significant in the energy transfer.

Both the underlying viscous fluid layer and the intervening air layer are also potential sites of energy dissipation. In a fluid film of thickness $h \ll R$, the flow can be described with lubrication theory. The pressure induced by the impact scales as $\rho V^{2}$, where $\rho$ is the density of the liquid drop, and it is approximately constant over the thickness. The corresponding pressure gradient $\sim \rho V^{2} / R$ beneath the drop generates a parabolic flow within the air layer and the viscous sublayer of characteristic velocity

$$
u \sim \frac{\rho V^{2}}{R} \frac{h^{2}}{\mu_{f}},
$$

where $\mu_{f}$ is the viscosity and $h$ the thickness of the relevant fluid layer (either the intervening air or the underlying oil). By conservation of mass, the thickness of the film decreases with a rate $\sim u h / R$, so during the impact (of duration $\sim \tau_{\sigma}$ ), the relative decrease in thickness $\Delta h / h$ scales as

$$
\frac{\Delta h}{h} \sim \frac{u \tau_{\sigma}}{R} \sim\left(\frac{h}{R}\right)^{2} W e \frac{\sqrt{\rho R \sigma}}{\mu_{f}} .
$$

Then, the energy dissipated by viscosity in the film is estimated

$$
\Delta E_{f} \sim h R^{2} \cdot \mu_{f}\left(\frac{u}{h}\right)^{2} \cdot \tau_{\sigma} \sim \frac{\rho^{5 / 2} h^{3} R^{3 / 2} V^{4}}{\mu_{f} \sigma^{1 / 2}} .
$$

This energy is compared to the kinetic energy of the drop at impact

$$
\frac{\Delta E_{f}}{\rho V^{2} R^{3}} \sim\left(\frac{h}{R}\right)^{3} W e \frac{\sqrt{\rho R \sigma}}{\mu_{f}} \sim \frac{h}{R} \cdot \frac{\Delta h}{h} .
$$

In our experiments, the Weber number never exceeds 20 . In the air layer $\left(\mu_{f}=\mu_{a} \simeq 1.8 \times 10^{-5}\right.$ $\mathrm{kg} / \mathrm{m} \cdot \mathrm{s}$ ), the ratio $\mu_{f} / \sqrt{\rho R \sigma}$ is of the order of $1.3 \times 10^{-4}$, so the air film is significantly thinned. Setting $\Delta h \sim h$ yields $h / R \sim 0.003$. As already mentioned, the critical thickness of the air layer required for the onset of coalescence is typically a few micrometers. Therefore, the corresponding energy dissipation in the air layer is of the order of $0.3 \%$ of the initial kinetic energy. On the other hand, in the underlying oil film, the viscosity is $10^{5} \mathrm{cSt}$, so the ratio $\mu_{f} / \sqrt{\rho R \sigma} \sim 700$. The initial 


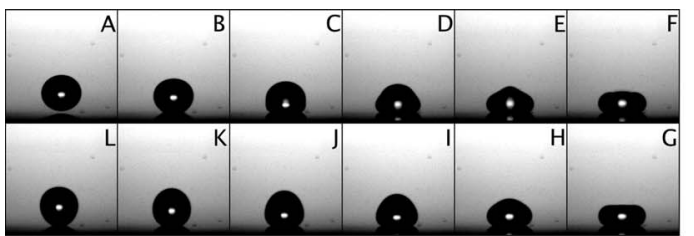

(a)

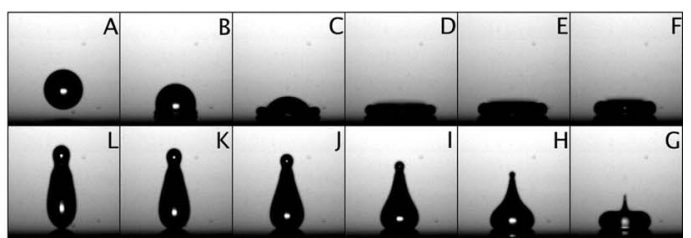

(b)

FIG. 17. Snapshots of the impacts corresponding to the experiments depicted in Fig. 14 [ $O h=0.02$, (a) We $=0.53$ and (b) $W e=11.1]$. Frames are separated by $1.33 \mathrm{~ms}$. Time is increasing from left to right on the first row, then from right to left on the second row, in order to reveal the strong time asymmetry at $W e=11.1$.

film thickness is less than $0.1 R$, so $\Delta h / h \lesssim 3 \times 10^{-4}$; the deformation of the liquid film is thus negligible, as is the corresponding energy dissipation.

It has been reported that shock waves may form when a water drop impacts a solid, in a manner reminiscent of the water-hammer effect. ${ }^{58,59}$ These shocks appear at the very early stages of impact, and are quickly dissipated thereafter. The timescale at which these shocks propagate and dissipate energy is $\tau_{s} \sim R / c \sim 1 \mu \mathrm{s}$, where $c \sim 1000 \mathrm{~m} / \mathrm{s}$ is the speed of sound in silicon oil. This timescale is several orders of magnitude smaller than the timescale (a few milliseconds) at which energy is dissipated according to Fig. 14(d). Moreover, impact is smoothed by the lubrication air layer that progressively raises the pressure at the bottom of the droplet. So shocks are also unlikely to contribute significantly to the energy loss observed in Fig. 14(d).

Another energy sink would be associated with the capillary waves traveling from the bottom to the top of the drop. ${ }^{46}$ We should first note that the droplet deformation is relatively symmetric in time at low We, while it is highly asymmetric at high We (Fig. 17). At low We [Fig. 17(a)], the droplet shape is very smooth, and only the spherical harmonics with $\ell \leq 3$ seem to be present. Conversely, at high We [Fig. 17(b)], a cusp appears in the droplet shape. This cusp, better seen in Fig. 18, moves upwards into the droplet as it moves downwards. Above the cusp, the shape is a spherical cap still moving at a speed $V$, which suggests that this part of the droplet is not yet aware of the impact: it has yet to be reached by the capillary waves. The cusp is then a kind of shock wave that appears when the impact velocity $V$ is larger than the velocity of the capillary waves $v_{\sigma}(\ell)$. On a sphere, this speed ratio is prescribed by

$$
\left(\frac{V}{v_{\sigma}(\ell)}\right)^{2}=\frac{4 W e}{\ell(\ell+2)(\ell-1)}
$$

which is larger than 1 for $W e=2$ when $\ell=2$. Unfortunately, the droplet is no longer a sphere, and the capillary waves are highly nonlinear-they doubtlessly interact with each other and exchange energy. Nevertheless, it is still likely that as We increases, progressively more wavenumbers $\ell$ become unstable and the cusp becomes sharper. This shock-like behavior might be responsible for the energy loss observed during the compression phase in Fig. 14(d). Nevertheless, further investigations of the internal flow are required before this question can be satisfactorily addressed. Since PIV experiments

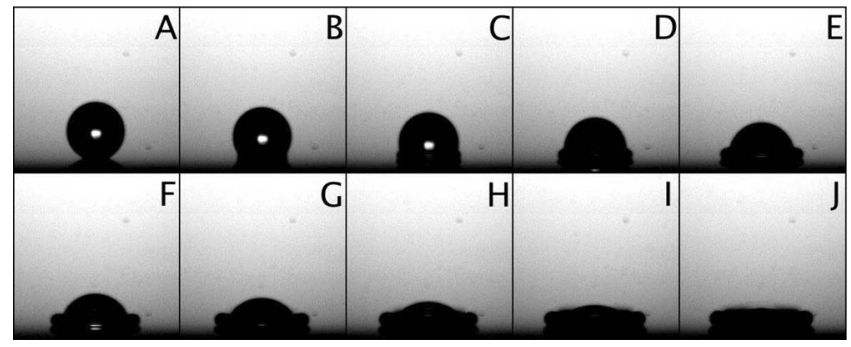

FIG. 18. Detail of the compression phase for the experiment depicted in Figs. 14(c) and 14(d) and Fig. 17(b) [Oh=0.02 and $W e=11.1]$. Frames are separated by $0.33 \mathrm{~ms}$. 
are still extremely challenging inside highly deformed millimetric droplets, numerical simulations such as those developed by Blanchette ${ }^{60,61}$ may provide a good alternative.

\section{v. CONCLUSION}

We have presented an experimental investigation of droplets impacting an inclined solid substrate coated with a thin layer of high viscosity liquid. The incoming drop may either completely or partially bounce or merge with the underlying liquid. Low viscosity drops are more likely to split into several satellite droplets. Through high-speed visualizations, we have described the sequence of events during impact as a function of the incident Weber number. The regime of complete rebound has been characterized by a series of black box parameters, including the contact time, the slip length, and the coefficients of restitution. Scaling laws based on theoretical arguments have been presented that successfully collapse data from experiments covering a broad range of parameters, including the Ohnesorge number and inclination angle. The coefficients of restitution are observed to be constant for $W e_{1 n}<2$ but to decrease significantly when $W e_{1 n}>2$. The additional loss of translational energy during rebound at high impact velocity has been further quantified by image processing. We have argued the probable importance of nonlinear capillary waves in this energy transfer, though the precise mechanism has yet to be determined and modeled.

\section{ACKNOWLEDGMENTS}

We thank the National Science Foundation (NSF) for financial support through Grant No. CBET-0966452. We gratefully acknowledge Jacy Bird, Lydia Bourouiba, and Ruben Rosales for fruitful discussions.

\section{APPENDIX: SCALING THE VAPOR THRUST IN LEIDENFROST BOUNCING}

Several studies have been performed on impacting Leidenfrost drops (e.g., Ref. 33). Although the impact dynamics appears similar to that reported here, the coefficients of restitution do not obey the same scaling laws. When a Leidenfrost droplet impacts a hot plate, a small amount of liquid is vaporized below the drop. This vapor ejection generates an upward thrust force on the droplet, in addition to the usual lubrication force. We here generalize the model of a stationary Leidenfrost drop $^{62}$ to the case of an impacting drop.

We assume that the gas layer below the drop has a uniform thickness $\delta(t)$. In the lubrication approximation, the horizontal (radial) velocity $u$ is parabolic in $z$, namely,

$$
u(r, z)=6 u_{m}(r)\left(\frac{z}{\delta}-\frac{z^{2}}{\delta^{2}}\right) \text { with } u_{m}=\frac{1}{\delta} \int_{0}^{\delta} u d z .
$$

The horizontal momentum equation yields

$$
\frac{d p}{d r}=-\frac{12 \mu_{v} u_{m}}{\delta^{2}}
$$

where $\mu_{v}$ is the viscosity of the vapor. The mean velocity $u_{m}$ in the film is inferred from the continuity equation

$$
\frac{1}{r} \partial_{r}(r u)+\partial_{z} v=0 \Rightarrow \frac{1}{r} \partial_{r}\left(r \delta u_{m}\right)+\dot{\delta}=v_{v a p} \Rightarrow u_{m}=\frac{r\left(v_{v a p}-\dot{\delta}\right)}{2 \delta},
$$

where $v_{\text {vap }}$ is the vapor velocity, namely, the relative speed at which the liquid/gas interface moves as the liquid is vaporized.

The thermal energy required to evaporate the liquid is provided by thermal conduction in the gas layer

$$
h_{l v} \rho_{v} v_{v a p}=-k_{v}\left(\frac{\partial T}{\partial z}\right)_{z=\delta} \Rightarrow v_{v a p}=\frac{k_{v}\left(T_{w}-T_{b}\right)}{\delta h_{l v} \rho_{v}}
$$


where $h_{l v}$ is the enthalpy of vaporization per unit mass, $\rho_{v}$ the vapor density, $k_{v}$ the vapor thermal conductivity, $T_{w}$ the solid wall temperature, and $T_{b}$ the temperature at the bottom of the drop. It is usual $^{62}$ to rewrite $v_{\text {vap }}$ as

$$
v_{v a p}=\frac{J a \alpha_{v}}{\delta},
$$

where $J a=c_{P}\left(T_{w}-T_{b}\right) / h_{l v}$ is the Jakob number, $c_{P}$ is the specific heat capacity of the vapor, and $\alpha_{v}=k_{v} /\left(\rho_{v} c_{P}\right)$ is the thermal diffusivity of the vapor.

The total vertical force applied by the gas film on the drop is obtained by integrating the momentum equation (A2) twice along $r$ and using (A3)

$$
F=2 \pi \int_{0}^{R}\left(p-p_{r=R}\right) r d r=\frac{3 \pi}{2} \frac{\mu_{v} R^{4}\left(J a \alpha_{v}-\delta \dot{\delta}\right)}{\delta^{4}} .
$$

The second term corresponds to the traditional lubrication force; it is proportional to $\dot{\delta} / \delta^{3}$ so it scales as $\mu_{v} R^{4} /\left(\delta^{2} \tau_{\sigma}\right)$. The first term is the vapor thrust.

When bouncing, a drop reverses its momentum; so if it impacts at speed $V$, it should experience a force that scales as $\rho R^{2} V^{2}$, where $\rho$ is the liquid density. Suppose first that vapor thrust is more important than the lubrication force. Then,

$$
\delta^{4} \sim \frac{\mu_{v} R^{2} J a \alpha_{v}}{\rho V^{2}} \Rightarrow \frac{J a \alpha_{v}}{\frac{\delta^{2}}{\tau_{\sigma}}} \sim \sqrt{\frac{\rho}{\rho_{v}} \frac{J a W e}{P r_{v}}},
$$

where $\operatorname{Pr}_{v}$ is the Prandlt number of the vapor. Conversely, if the lubrication force dominates vapor thrust

$$
\delta^{2} \sim \frac{\mu_{v} R^{2}}{\rho V^{2} \tau_{\sigma}} \Rightarrow \frac{J a \alpha_{v}}{\frac{\delta^{2}}{\tau_{\sigma}}} \sim \frac{\rho}{\rho_{v}} \frac{J a W e}{P r_{v}} .
$$

In both cases, the importance of vapor thrust over lubrication is prescribed by the ratio $(\rho J a W e) /\left(\rho_{v} P r_{v}\right)$. For water, $h_{l v}=2.2 \times 10^{6} \mathrm{~J} / \mathrm{kg}, \mu_{v}=12.55 \times 10^{-6} \mathrm{~kg} / \mathrm{m} \cdot \mathrm{s}, k_{v}=0.025 \mathrm{~W} / \mathrm{m} \mathrm{K}$, $\rho=1000 \mathrm{~kg} / \mathrm{m}^{3}$ and $\rho_{v}=0.597 \mathrm{~kg} / \mathrm{m}^{3}$. If $T_{w}-T_{b}=200 \mathrm{~K}$, we obtain

$$
\frac{\rho J a W e}{\rho_{v} P r_{v}} \sim 300 W e
$$

which exceeds unity provided $W e>0.003$.

We conclude that, in the experiments of Biance et al. ${ }^{33}$ vapor thrust should have a significant influence on the impact dynamics by providing an additional force upwards which is larger than the usual lubrication force. This may serve to rationalize why the normal coefficient of restitution decreases more sharply with $W e$ in the Leidenfrost configuration (the importance of vapor thrust increasing with $\mathrm{We}$ ). Moreover, coefficients of restitution approaching unity (over 1000 successive impacts) have been reported in this Leidenfrost case at low We. We note that, if the coefficient of restitution happened to be slightly less than unity (e.g., $e_{n}=0.99$ ), the impact velocity would be reduced by a factor $0.99^{1000} \simeq 4 \times 10^{-5}$. Take-off would then be impossible to observe. We therefore suspect that in the low We limit of the Leidenfrost impact, vapor thrust balances dissipation in the long term and yields an average normal coefficient of restitution of 1.

${ }^{1}$ A. Yarin, "Drop impact dynamics: Splashing, spreading, receding, bouncing...” Annu. Rev. Fluid Mech. 38, 159 (2006).

${ }^{2}$ L. Bourouiba and J. W. M. Bush, "Drops and bubbles," Handbook of Environmental Fluid Dynamics (Taylor \& Francis, 2012), Vol. 1.

${ }^{3}$ X. Yang, L. V. Madden, D. L. Reichard, R. D. Fox, and M. A. Ellis, "Motion analysis of drop impaction on a strawberry surface," Agric. Forest Meteorol. 56, 67 (1991).

${ }^{4}$ V. Bergeron, D. Bonn, J.-Y. Martin, and L. Vovelle, "Controlling droplet deposition with polymer additives," Nature (London) 405, 772 (2000).

${ }^{5}$ R.-H. Chen, S.-L. Chiu, and T.-H. Lin, "On the collision behaviors of a diesel drop impinging on a hot surface," Exp. Therm. Fluid Sci. 32, 587 (2007).

${ }^{6}$ S. Sinha-Ray, Y. Zhang, and A. L. Yarin, "Thorny devil nanotextured fibers: the way to cooling rates on the order of $1 \mathrm{kw} / \mathrm{cm}^{2}$," Langmuir 27, 215 (2011). 
${ }^{7}$ D. B. van Dam and C. Le Clerc, "Experimental study of the impact of an ink-jet printed droplet on a solid substrate," Phys. Fluids 16, 3403 (2004).

${ }^{8}$ R. Blossey, "Self-cleaning surfaces: virtual realities," Nature Mater. 2, 301 (2003).

${ }^{9}$ J. W. M. Bush, "Quantum mechanics writ large," Proc. Natl. Acad. Sci. U.S.A. 107, 17455 (2010).

${ }^{10}$ Y. Couder and E. Fort, "Single-particle diffraction and interference at a macroscopic scale," Phys. Rev. Lett. 97, 154101 (2006).

${ }^{11}$ E. Fort, A. Eddi, A. Boudaoud, J. Moukhtar, and Y. Couder, "Path-memory induced quantization of classical orbits," Proc. Natl. Acad. Sci. U.S.A. 107, 17515 (2010).

${ }^{12}$ A. Eddi, E. Fort, F. Moisy, and Y. Couder, "Unpredictable tunneling of a classical wave-particle association," Phys. Rev. Lett. 102, 240401 (2009).

${ }^{13}$ L. Rayleigh, "Further observations upon liquid jets, in continuation of those recorded in the Royal Society's Proceedings for March and May, 1879," Proc. R. Soc. London, Ser. A 34, 130 (1882).

${ }^{14}$ J. Qian and C. K. Law, "Regimes of coalescence and separation in droplet collision," J. Fluid Mech. 331, 59 (1997).

${ }^{15}$ G. Brenn, D. Valkovska, and K. D. Danov, "The formation of satellite droplets by unstable binary drop collisions," Phys. Fluids 13, 2463 (2001).

${ }^{16}$ K. L. Pan and C. K. Law, "Dynamics of droplet-film collision,” J. Fluid Mech. 587, 1 (2007).

${ }^{17}$ P. Dell'Aversana, V. Tontodonato, and L. Carotenuto, "Suppression of coalescence and of wetting: The shape of the interstitial film," Phys. Fluids 9, 2475 (1997).

${ }^{18}$ D. Terwagne, N. Vandewalle, and S. Dorbolo, "Lifetime of a bouncing droplet," Phys. Rev. E 76, 056311 (2007).

${ }^{19}$ O. Reynolds, "On the floating of drops on the surface of water depending only on the purity of the surface," Proc. Lit. Phil. Soc. Manchester 21, 1 (1881).

${ }^{20}$ D. Richard, C. Clanet, and D. Quéré, "Contact time of a bouncing drop," Nature (London) 417, 811 (2002).

${ }^{21}$ K. Okumura, F. Chevy, D. Richard, D. Quéré, and C. Clanet, "Water spring: A model for bouncing drops," Europhys. Lett. 62, 237 (2003).

${ }^{22}$ G. E. Charles and S. G. Mason, "The coalescence of liquid drops with flat liquid/liquid interfaces," J. Colloid Sci. 15, 236 (1960).

${ }^{23}$ I. B. Ivanov and T. T. Traykov, "Hydrodynamics of thin liquid films: Rate of thinning of emulsion thin films of pure liquids," Int. J. Multiphase Flow 2, 397 (1976).

${ }^{24}$ Y. He, T. Howes, J. D. Litster, and G. H. Ko, "Experimental study of drop-interface coalescence in the presence of polymer stabilisers," Colloids Surf., A 207, 89 (2002).

${ }^{25}$ T. D. Hodgson and J. C. Lee, "The effect of surfactants on the coalescence of a drop at an interface," J. Colloid Interface Sci. 30, 94 (1969).

${ }^{26}$ Y. Amarouchene, G. Cristobal, and H. Kellay, "Noncoalescing drops," Phys. Rev. Lett. 87, 206104 (2001).

${ }^{27}$ G. P. Neitzel and P. Dell'Aversana, "Noncoalescence and nonwetting behavior of liquids," Annu. Rev. Fluid Mech. 34, 267 (2002).

${ }^{28}$ Y. Cai, "Phenomena of a liquid drop falling to a liquid surface," Exp. Fluids 7, 388 (1989).

${ }^{29}$ P. Dell'Aversana, J. R. Banavar, and J. Koplik, "Suppression of coalescence by shear and temperature gradients," Phys. Fluids 8, 15 (1996).

${ }^{30}$ K. R. Sreenivas, P. K. De, and J. H. Arakeri, "Levitation of a drop over a film flow," J. Fluid Mech. 380, 297 (1999).

${ }^{31}$ M. Thrasher, S. Jung, Y. K. Pang, C.-P. Chuu, and H. L. Swinney, "Bouncing jet: A Newtonian liquid rebounding off a free surface," Phys. Rev. E 76, 056319 (2007).

${ }^{32}$ A.-L. Biance, C. Clanet, and D. Quéré, "Leidenfrost drops," Phys. Fluids 15, 1632 (2003).

${ }^{33}$ A.-L. Biance, F. Chévy, C. Clanet, G. Lagubeau, and D. Quéré, "On the elasticity of an inertial liquid shock," J. Fluid Mech. 554, 47 (2006).

${ }^{34}$ B. Derjaguin and P. Prokhorov, "On the cause of noncoalescence of liquid drops upon impact," Prog. Surf. Sci. 43, 273 (1993).

${ }^{35}$ Y. Couder, E. Fort, C.-H. Gautier, and A. Boudaoud, "From bouncing to floating: Noncoalescence of drops on a fluid bath," Phys. Rev. Lett. 94, 177801 (2005).

${ }^{36}$ T. Gilet, D. Terwagne, N. Vandewalle, and S. Dorbolo, "Dynamics of a bouncing droplet onto a vertically vibrated interface," Phys. Rev. Lett. 100, 167802 (2008).

${ }^{37}$ T. Gilet and J. W. M. Bush, "Chaotic bouncing of a droplet on a soap film," Phys. Rev. Lett. 102, 014501 (2009).

${ }^{38}$ T. Gilet, N. Vandewalle, and S. Dorbolo, "Completely inelastic ball," Phys. Rev. E 79, 055201 (2009).

${ }^{39}$ O. W. Jayaratne and B. J. Mason, "The coalescence and bouncing of water drops at an air/water interface," Proc. R. Soc. London, Ser. A 280, 545 (1964).

${ }^{40}$ T. Gilet and J. W. M. Bush, "The fluid trampoline: Droplets bouncing on a soap film," J. Fluid Mech. 625, 167 (2009).

${ }^{41}$ D. Richard and D. Quéré, "Bouncing water drops," Europhys. Lett. 50, 769 (2000).

${ }^{42}$ P. Aussillous and D. Quéré, "Properties of liquid marbles," Proc. R. Soc. London, Ser. A 462, 973 (2006).

${ }^{43}$ W. J. Stronge and A. D. C. Ashcroft, "Oblique impact of inflated balls at large deflections," Int. J. Impact Eng. 34, 1003 (2007).

${ }^{44}$ D. Terwagne, T. Gilet, N. Vandewalle, and S. Dorbolo, "From a bouncing compound drop to a double emulsion," Langmuir 26, 11680 (2010).

${ }^{45}$ D. Bartolo, C. Josserand, and D. Bonn, "Singular jets and bubbles in drop impact," Phys. Rev. Lett. 96, 124501 (2006).

${ }^{46}$ Y. Renardy, S. Popinet, L. Duchemin, M. Renardy, S. Zaleski, C. Josserand, M. A. Drumright-Clarke, D. Richard, C. Clanet, and D. Quéré, "Pyramidal and toroidal water drops after impact on a solid surface," J. Fluid Mech. 484, 69 (2003).

${ }^{47}$ F. Blanchette and T. P. Bigioni, "Partial coalescence of drops at liquid interfaces," Nat. Phys. 2, 1 (2006).

${ }^{48}$ T. Gilet, K. Mulleners, J. P. Lecomte, N. Vandewalle, and S. Dorbolo, "Critical parameters for the partial coalescence of a droplet," Phys. Rev. E 75, 036303 (2007). 
${ }^{49}$ K. Anders, N. Roth, and A. Frohn, "The velocity change of ethanol droplets during collision with a wall analysed by image processing," Exp. Fluids 15, 91 (1993).

${ }^{50}$ R. Cross, "Grip-slip behavior of a bouncing ball,” Am. J. Phys. 70, 1093 (2002).

${ }^{51}$ C.-Y. Wu, C. Thornton, and L.-Y. Li, "A semi-analytical model for oblique impacts of elastoplastic spheres," Proc. R. Soc. London, Ser. A 465, 937 (2009).

${ }^{52}$ A. A. Kantak and R. H. Davis, "Elastohydrodynamic theory for wet oblique collisions," Powder Technol. 168, 42 (2006)

${ }^{53}$ A. A. Kantak and R. H. Davis, "Oblique collisions and rebound of spheres from a wetted surface," J. Fluid Mech. 509, 63 (2004).

${ }^{54}$ M. Reyssat, F. Pardo, and D. Quéré, “Drops onto gradients of texture,” Europhys. Lett. 87, 36003 (2009).

${ }^{55}$ A.-L. Biance, C. Pirat, and C. Ybert, "Drop fragmentation due to hole formation during Leidenfrost impact," Phys. Fluids 23, 022104 (2011)

${ }^{56}$ C. Clanet, C. Béguin, D. Richard, and D. Quéré, "Maximal deformation of an impacting drop," J. Fluid Mech. 517, 199 (2004).

${ }^{57}$ J. Eggers, M. A. Fontelos, C. Josserand, and S. Zaleski, "Drop dynamics after impact on a solid wall: Theory and simulations," Phys. Fluids 22, 062101 (2010).

${ }^{58}$ J. Field, "Liquid impact: theory, experiment, applications," Wear 233, 1 (1999).

${ }^{59}$ D. Obreschkow, N. Dorsaz, P. Kobel, A. de Bosset, M. Tinguely, J. Field, and M. Farhat, "Confined shocks inside isolated liquid volumes: A new path of erosion?” Phys. Fluids 23, 101702 (2011).

${ }^{60}$ F. Blanchette and T. P. Bigioni, "Dynamics of drop coalescence at fluid interfaces," J. Fluid Mech. 620, 333 (2009).

${ }^{61}$ F. Blanchette, L. Messio, and J. W. M. Bush, "The influence of surface tension gradients on drop coalescence," Phys. Fluids 21, 072107 (2009).

${ }^{62}$ V. Carey, Liquid-Vapor Phase-Change Phenomena, 2nd ed. (Taylor \& Francis, 2008).

${ }^{63}$ J. G. Leidenfrost, De Aquae Communis Nonnullis Qualitatibus Tractatus (Duisburg, 1756). 